\title{
Early loss of mammalian target of rapamycin complex 1 (mTORC1) signalling and reduction in cell size during dominant-negative suppression of hepatic nuclear factor 1- $\alpha$ (HNF1A) function in INS-1 insulinoma cells
}

\author{
A. M. Farrelly $\cdot$ H. Wobser $\cdot$ C. Bonner $\cdot$ S. Anguissola $\cdot$ \\ M. Rehm • C. G. Concannon • J. H. M. Prehn • \\ M. M. Byrne
}

Received: 23 May 2008 / Accepted: 7 September 2008 / Published online: 24 October 2008

(C) Springer-Verlag 2008

\begin{abstract}
Aims/hypothesis Mutations in the HNF1A (previously known as TCF1) gene encoding hepatocyte nuclear factor- $1 \alpha$ (HNF1A) lead to the development of maturity-onset diabetes of the young, type 3 (HNF1A-MODY), characterised by impaired insulin secretion and a reduction in beta cell mass. HNF1A plays an important role in pancreatic beta cell differentiation and survival. The mammalian target of rapamycin (mTOR) is a central growth factor- and nutrientactivated protein kinase controlling cell metabolism, growth and survival. We investigated the role of mTOR inactivation in the decline in beta cell mass in a cellular model of HNF1A-MODY.

Methods Previously we showed that suppression of HNF1A function via expression of a dominant-negative mutant (DN-HNF1A) decreases insulin gene transcription in insulinoma (INS-1) cells. We investigated the signalling of two distinct mTOR protein complexes, mTORC1 and mTORC2, in response to DN-HNF1A induction.
\end{abstract}

Electronic supplementary material The online version of this article (doi:10.1007/s00125-008-1168-8) contains supplementary material, which is available to authorised users.

A. M. Farrelly $\cdot$ M. M. Byrne $(\bowtie)$

Department of Endocrinology,

Mater Misericordiae University Hospital,

Eccles Street,

Dublin 7, Ireland

e-mail: mbyrne@mater.ie

H. Wobser $\cdot$ C. Bonner $\cdot$ S. Anguissola $\cdot$ M. Rehm •

C. G. Concannon J J. H. M. Prehn

Department of Physiology and Medical Physics,

Royal College of Surgeons in Ireland,

Dublin 2, Ireland
Results We observed delayed inactivation of mTORC2 $48 \mathrm{~h}$ after DN-HNF1A induction, evidenced by a reduction in serine 473 phosphorylation of thymoma viral protooncogene 1 (AKT1). We also observed an early inactivation of mTORC1 $24 \mathrm{~h}$ after DN-HNF1A induction, which was detected by decreases in threonine 389 phosphorylation of p70 ribosomal protein S6 kinase (S6K1) and serine 65 phosphorylation of translational inhibitor eukaryotic translation initiation factor 4E binding protein 1 (4E-BP1). Flow cytometry and gene expression analysis demonstrated a preapoptotic decrease in INS-1 cell size in response to DNHNF1A induction, and an increase in the level of the mTORC1-regulated cell-cycle inhibitor, cyclin-dependent kinase inhibitor 1B p27.

Conclusions/interpretation Our data suggest that mTOR kinase and signalling through $\mathrm{mTORC} 1$ are highly sensitive to suppression of HNF1A function, and may contribute to disturbance of cell-size regulation and cell-cycle progression in HNF1A-MODY.

Keywords AKT1 - Beta cell mass · Cell size - HNF1A . INS-1 $\cdot$ MODY $\cdot$ mTOR $\cdot$ S6K1

\begin{tabular}{|c|c|}
\hline \multicolumn{2}{|c|}{ Abbreviations } \\
\hline 4E-BP1 & $\begin{array}{l}\text { eukaryotic translation initiation factor } 4 \mathrm{E} \\
\text { binding protein } 1\end{array}$ \\
\hline AKT1 & thymoma viral proto-oncogene 1 \\
\hline DN & dominant-negative \\
\hline EIF4E & eukaryotic translation initiation factor $4 \mathrm{E}$ \\
\hline HNF1A & hepatocyte nuclear factor- $1 \alpha$ \\
\hline INS-1 & insulinoma cell line \\
\hline GBL & $\mathrm{G}$ protein $\beta$ subunit-like \\
\hline
\end{tabular}


mTOR mammalian target of rapamycin

mTORC1 mammalian target of rapamycin complex 1

mTORC2 mammalian target of rapamycin complex 2

p27 cyclin-dependent kinase inhibitor 1B

PDK1 phosphoinositide-dependent kinase-1

PI3K phosphatidylinositol 3-kinase

PRAS40 proline-rich Akt substrate of $40 \mathrm{kDa}$

qPCR real-time quantitative PCR

Raptor regulator-associated protein of mTOR

RHEB RAS-homologue enriched in brain

Rictor rapamycin-insensitive companion of mTOR

S6K1 ribosomal protein S6 kinase

SIN1 stress-activated map kinase-interacting protein 1

TSC1 tuberous sclerosis 1 protein

$\mathrm{TSC} 2$ tuberous sclerosis 2 protein

WT wild type

\section{Introduction}

MODY is an autosomal dominant and monogenic form of diabetes characterised by early age of onset and primary pancreatic beta cell dysfunction [1]. Hepatocyte nuclear factor- $1 \alpha(\mathrm{HNF} 1 \mathrm{~A})$ MODY is believed to be the commonest form of MODY [2] and results from heterozygous loss-of-function mutations of transcription factor $H N F 1 \mathrm{~A}$ (previously known as TCF1) [3, 4]. HNF1A is produced in the liver, kidney, intestine and pancreatic islets [5]. It participates in a transcription factor network that regulates pancreatic development as well as fatty acid, protein and carbohydrate metabolism [6]. This network has also been shown to be critically involved in the pathophysiology of type 2 diabetes [7].

Beta cell transgenic mice carrying a deletion of the Hnfla gene have defective glucose-stimulated insulin secretion without insulin resistance of target tissues [8], similar to individuals with HNF1A-MODY [9]. Animal models of HNF1A-MODY suggest a decline in functional beta cell mass as the key underlying mechanism of this defect [10]. A decrease in beta cell mass can be caused by defects in beta cell differentiation and proliferation, a decrease in beta cell size or increased beta cell apoptosis. Previous studies from our and other laboratories have shown that knockdown of Hnfla or expression of dominantnegative mutants of Hnfla (DN-HNF1A) decreases insulin release and insulin gene transcription, and inhibits the activation of the phosphoinositide 3-kinase (PI3K)/AKT1 pathway, thereby arresting cell proliferation and sensitising beta cells to apoptosis [11-13].

Reduced insulin signalling can also impact on mammalian target of rapamycin (mTOR) signalling. Originally identified in Saccharomyces cerevisiae [14], mTORs are PI3K-related
Ser/Thr kinases. mTOR integrates signals from a variety of upstream sources, including nutrients such as amino acids and mitogenic and growth factors [15]. The signalling pathway initiated by growth factors involves insulin/IGF receptor-induced PI3K and AKT1 activation leading to phosphorylation of tuberous sclerosis 1 protein (TSC1)/ tuberous sclerosis 2 protein (TSC2) and inhibition of RAShomologue enriched in brain (RHEB)-GTPase [16]. mTOR complex 1 (mTORC1) and mTOR complex 2 (mTORC2) are two structurally and functionally distinct protein complexes that share mTOR as their catalytic subunit. mTORC1 contains mTOR, regulator-associated protein of mTOR (Raptor), proline-rich Akt substrate of $40 \mathrm{kDa}$ (PRAS40) and $G$ protein $\beta$ subunit-like protein (GBL) and regulates temporal aspects of cell growth, such as ribosomal biogenesis and protein synthesis. Activated mTORC1 directly phosphorylates ribosomal p70 S6 kinase (S6K1), leading to its activation and phosphorylation of the repressors of mRNA translation 4E-binding proteins (4E-BP), thereby causing their inactivation [17]. mTORC1 initiates a negative feedback loop to modify the activity of AKT1 through S6K1. mTORC1activated kinase S6K1 phosphorylates IRS1 and IRS2 proteins, resulting in decreased IRS-protein stability in insulin/ insulin-like growth factor-responsive cells [17]. Another mTOR complex, mTORC2, contains mTOR, rapamycininsensitive companion of mTOR (Rictor), PRR5/prolinerich protein 5 (Protor), mammalian stress-activated protein kinase-interacting protein 1 (SIN1) and GBL, and appears to regulate long-term changes in cellular physiology such as spatial organisation [18]. mTORC2 also phosphorylates and activates AKT1 at the stimulatory Ser473 residue. In conjunction with phosphoinositide-dependent kinase-1 (PDK1)mediated phosphorylation of $\mathrm{Thr} 308$, this modification drives the full activation of AKT1 $[19,20]$. The TSC1TSC2 complex was found to physically associate with mTORC2 and may regulate the mTORC2 phosphorylation of AKT1 [21].

Given the impact of dominant-negative suppression of HNF1A function on insulin signalling and the central role of mTOR in mediating growth-factor signalling, we investigated the potential role of mTOR in the decline in beta cell mass in HNF1A-MODY, using the in vitro model of INS-1 cells inducibly expressing DN-HNF1A.

\section{Methods}

Cultivation and treatment of insulinoma INS-1 cells overexpressing Hnfla in an inducible system Rat INS-1 insulinoma cells overexpressing wild-type Hnfla (WTHNF1A) or a dominant-negative mutant of Hnfla (DNHNF1A) (SM6) under control of a doxycycline-dependent transcriptional activator have been described previously 
$[13,22]$. The SM6 mutant contains a substitution of 83 amino acids in the Hnfla DNA-binding domain, resulting in the formation of non-functional heterodimers with endogenous HNF1A [23] (see Electronic supplementary material $[\mathrm{ESM}])$.

Western blotting Cells were rinsed with ice-cold PBS and lysed in buffer containing $62.5 \mathrm{mmol} / \mathrm{l}$ Tris- $\mathrm{HCl}$ (pH 6.8), $2 \%$ SDS (wt/vol.), $10 \%$ glycerin (wt/vol.) and protease inhibitor cocktail. Protein content was determined using the Pierce BCA Micro Protein Assay kit (Rockford, IL, USA). Samples were supplemented with 2-mercaptoethanol and denatured at $95^{\circ} \mathrm{C}$ for $5 \mathrm{~min}$. An equal amount of protein (20-50 $\mu \mathrm{g}$ ) was separated with 5-15\% SDS-PAGE (wt/vol.) and blotted to nitrocellulose membranes (Protean BA 85; Schleicher \& Schuell, Dassel, Germany). The blots were blocked with $5 \%$ non-fat milk (wt/vol.) in blocking solution (15 mmol/l Tris- $\mathrm{HCl}$ [pH 7.5], $200 \mathrm{mmol} / \mathrm{l} \mathrm{NaCl}$ and $0.1 \%$ Tween-20 [wt/vol.]) for $2 \mathrm{~h}$ at room temperature. Membranes were incubated overnight at $4^{\circ} \mathrm{C}$ with the primary antibodies (see ESM).

Flow cytometry After DN-HNF1A induction, INS-1 cells were collected with trypsin-EDTA and incubated in binding buffer $(10 \mathrm{mmol} / \mathrm{l}$ HEPES, $135 \mathrm{mmol} / \mathrm{l} \mathrm{NaCl}, 5 \mathrm{~mm} / \mathrm{l}$ $\left.\mathrm{CaCl}_{2}\right)$ containing Annexin-V FITC $(5 \mu \mathrm{l} / \mathrm{ml})$ (BioVision, Mountain View, CA, USA) and propidium iodide $(1 \mu \mathrm{g} / \mathrm{ml})$ for $15 \mathrm{~min}$ at $37^{\circ} \mathrm{C}$. Cells $\left(1 \times 10^{5}\right)$ were resuspended in icecold binding buffer and analysed on a Cyflow ML 16 flow cytometer (Partec, Münster, Germany) equipped with a 488, 405 and $532 \mathrm{~nm}$ laser. Annexin- $\mathrm{V}$ was excited with the $488 \mathrm{~nm}$ laser and fluorescence emission was collected in the FL1 channel through a $520 \mathrm{~nm}$ band pass filter; propidium iodide was excited with the $488 \mathrm{~nm}$ laser and fluorescence emission was collected in the FL3 channel through a $620 \mathrm{~nm}$ long pass filter. Gated cells $\left(1 \times 10^{4}\right)$ were acquired for each sample and analysed by using the Flowmax software (Partec, Münster, Germany). Cell-size analysis was performed on healthy cells gated on a forward scatter channel/FL1 scatter plot; apoptotic AnnexinV-positive and necrotic propidium iodide-positive cells were therefore excluded from the analysis, and the median values of the forward scatter of the remaining populations used a measure of cell size.

Real-time quantitative PCR Expression of Raptor Rictor, Sin1 (also known as Mapkap1), mTor (also known as Frap1), cyclin-dependent kinase inhibitor 1B (p27 [also known as $C d k n 1 b])$ and L-type pyruvate kinase $(P k l r)$ mRNA was examined using real-time quantitative PCR (qPCR). INS-1 cells were harvested at the appropriate timepoints and total RNA was extracted using the RNeasy mini Kit (Qiagen, Crawley, UK). First-strand cDNA synthesis was performed with $2 \mu \mathrm{g}$ total RNA as template using Superscript II reverse transcriptase (Invitrogen, Paisley, UK) primed with 50 pmol random hexamers (New England Biolabs, Ipswich, MA, USA). qPCR was performed using the LightCycler 2.0 (Roche Diagnostics, Indianapolis, IN, USA) and the QuantiTech SYBR Green PCR kit (Qiagen) as per manufacturers' protocols and 25 pmol of primer pair concentration (Sigma-Genosys, Zwÿndrecht, the Netherlands). The PCR products were designed to be $150-200 \mathrm{bp}$ in length. Specific primers for each gene analysed were designed using Primer3 software (http://frodo.wi.mit.edu/ cgi-bin/ primer3/primer3_http://www.cgi; see ESM) [24]. Each primer pair was tested with a logarithmic dilution of a cDNA mix to generate a linear standard curve which was used to calculate the PCR efficiency and their specificity was determined by melting-curve analysis and gel electrophoresis of products. The PCR reactions were performed in a $20 \mu \mathrm{l}$ volume. The PCR was performed as follows: there was an activation step of HotStartTaq polymerase at $95^{\circ} \mathrm{C}$ for $15 \mathrm{~min}$, followed by 50 cycles of denaturation at $95^{\circ} \mathrm{C}$ for $15 \mathrm{~s}$, annealing at $59^{\circ} \mathrm{C}$ for $20 \mathrm{~s}$, and extension at $72^{\circ} \mathrm{C}$ for $20 \mathrm{~s}$. The data were analysed using Lightcycler Software 4.0 with all samples normalised to $\beta$-actin.

Statistics Data are given as means \pm SE. For statistical comparison, ANOVA and subsequent Tukey's test were employed. Results were considered to be statistically significant where $p<0.05$.

\section{Results}

INS-1 cells inducibly expressing DN-HNF1A but not WTHNF1A show an early decrease in Thr389 S6K1 phosphorylation Expression of DN-HNF1A was induced in INS-1 cells by treatment with doxycycline for 12, 24, 36 and $48 \mathrm{~h}$. Treatment with $500 \mathrm{ng} / \mathrm{ml}$ doxycycline led to a rapid induction of DN-HNF1A in INS-1 cells stably transfected with the respective doxycycline-dependent transcriptional activator (Fig. 1a). To explore alterations in mTOR signalling, the phosphorylation status of AKT1 (Ser473) and S6K1 (Thr389) was analysed. In agreement with our previous study [12], we found that induction of DNHNF1A reduced mTORC2-mediated Ser473 phosphorylation of AKT1 after $48 \mathrm{~h}$ (see also Fig. 4c). This reduction in AKT1 Ser473 phosphorylation correlated with an increase in caspase- 3 activity and apoptotic cell death in the cultures [12]. In contrast, mTORC1-dependent phosphorylation of S6K1 within the linker domain ( $\mathrm{Thr} 389)$ was significantly reduced as early as $24 \mathrm{~h}$ after induction of DN-HNF1A (Fig. 1b). This decrease persisted during the $48 \mathrm{~h}$ of DNHNF1A induction. Dephosphorylation of S6K1 at Thr389 
a

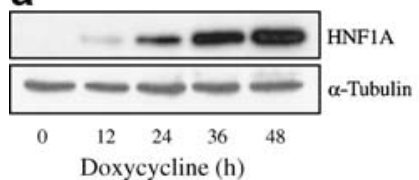

b
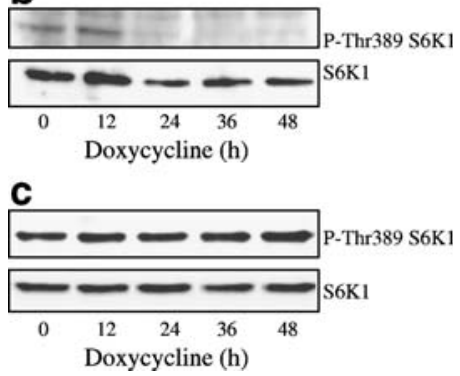

Fig. 1 DN-HNF1A induces an early decrease in mTORC1 signalling. a Time course of induction of DN-HNF1A in response to $500 \mathrm{ng} / \mathrm{ml}$ doxycycline. Immunodetection was performed using a HNF1A antibody. INS-1 cells were induced to overproduce DN-HNF1A (b) or WT-HNF1A (c) for 12, 24, 36 and $48 \mathrm{~h}$. The activation status of p70 S6 kinase was detected by western blotting using a Thr389phosphorylation-specific antibody, designated by P-Thr389. The nitrocellulose membrane was stripped and re-probed for total S6K1. Experiments were repeated twice with similar results

could not be detected in cells induced to produce WTHNF1A (Fig. 1c). These results suggested a differential inactivation of mTORC1- and mTORC2-dependent signalling in response to DN-HNF1A expression.

Early inactivation of mTORC1- and S6K1-dependent signalling pathways in response to DN-HNF1A To further explore this finding we analysed the activation status of other mTORC1- and S6K1-dependent signalling pathways in response to DN-HNF1A expression. mTORC1 controls protein synthesis via phosphorylation of 4E-BP1 [25]. 4EBP1 normally binds to and inhibits eukaryotic initiation factor 4 (eIF4E), thereby blocking cap-dependent translation initiation. INS-1 cells were induced to express DNHNF1A over $48 \mathrm{~h}$. Similar to the effects we observed regarding the activation status of S6K1, we observed a decrease in Ser65 phosphorylation of 4E-BP1 as early as $24 \mathrm{~h}$ after the induction of DN-HNF1A (Fig. 2a).

We also investigated S6K1-dependent phosphorylation of mTOR. S6K1 is able to phosphorylate mTOR at Ser2448, a mechanism described as a feed-forward activation loop [26]. Using a phosphorylation-specific antibody we detected a reduction in Ser2448 mTOR phosphorylation $24 \mathrm{~h}$ after DN-HNF1A induction that correlated with the decrease in phosphorylation (Fig. 2b). Total protein levels of mTOR remained stable during the induction of DNHNF1A. In contrast to the effects observed upon DNHNF1A induction, WT-HNF1A INS-1 cells failed to exhibit any decrease in mTOR Ser2448 phosphorylation (data not shown).

Differential gene expression of mTorc1 and mTorc2 constituent's Raptor, Rictor and Sin1 does not occur during the induction of DN-HNF1A The differential effect of DNHNF1A on mTORC1- and mTORC2-dependent signal transduction could be secondary to changes in gene expression of individual $m$ Torc 1 and $m$ Torc 2 constituents. While $m T O R$ and $G b l$ are components of both mTorcl and mTorc, changes in Raptor, Rictor and Sin1 gene expression upon induction of DN-HNF1A could manifest in differential mTORC activities. To address this question, cells were induced to express Hnfla and RNA was isolated between 8 and $48 \mathrm{~h}$ following induction. Gene expression was analysed by qPCR relative to the expression of $\beta$-actin mRNA, which did not change over $48 \mathrm{~h}$ (data not shown). Control experiments detected a prominent reduction in mRNA levels of Pklr, a known Hnfla target gene, in response to DN-HNF1A induction (Fig. 3a). Although we detected increased levels over time, we failed to detect any significant difference in expression levels of Raptor (constituent of $m$ Torcl) compared with Rictor or Sin 1 mRNA (constituents of $m$ Torc2 2 ), suggesting that alterations in gene expression did not cause the differential inactivation kinetics of mTORC1 and mTORC2 (Fig. 3b-d). Levels of $m$ Tor mRNA remained largely unaffected by DN-HNF1A induction (Fig. 3e).

Effect of extracellular glucose on mTORC1-dependent S6K1 and mTORC2-dependent AKT1 phosphorylation mTORC1 signalling is dependent on nutrient and energy supply, whereas comparatively little is known about the upstream regulation of $\mathrm{mTORC} 2[18,21]$. To explore the

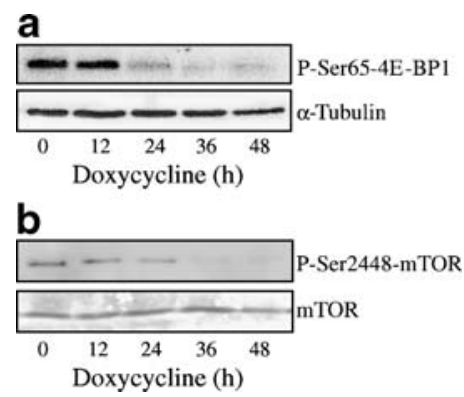

Fig. 2 Early decrease in mTORC1-mediated 4E-BP1 phosphorylation and S6K1-mediated mTOR phosphorylation. INS-1 cells were induced to overproduce DN-HNF1A for $0,12,24,36$ and $48 \mathrm{~h}$. a The activation status of 4E-BP1 was detected by western blotting using a Ser65-phosphorylation-specific antibody (P-Ser65). Membrane was stripped and re-probed with an anti- $\alpha$ tubulin antibody to prove equal loading of samples. b The activation of mTOR was detected by western blotting using a Ser2448 phosphorylation antibody (P-Ser2448). The experiment was repeated four times with similar results 
effect of nutrient supply on the differential inactivation of mTORC1- and mTORC2-dependent signalling in response to DN-HNF1A, we investigated the effect of extracellular glucose levels on the Thr389 phosphorylation status of S6K1 and the Ser473 phosphorylation status of AKT1. INS-1 cells were induced to express DN-HNF-1A for 24 or $48 \mathrm{~h}$ at a normal glucose concentration of $6 \mathrm{mmol} / \mathrm{l}$, and were subsequently challenged with increasing glucose concentrations for $30 \mathrm{~min}$. In agreement with previous reports, non-induced controls showed a glucose-dependent Thr389 phosphorylation of S6K1 (Fig. 4a). After induction of DN-HNF1A, the overall Thr389 phosphorylation of S6K1 was significantly reduced at all glucose concentrations tested (Fig. 4a). However, INS-1 cells expressing DNHNF1A were still glucose responsive, as a 30 min treatment in $18 \mathrm{mmol} / \mathrm{l}$ extracellular glucose elevated the levels of Thr389 phosphorylated S6K1 to levels detectable in noninduced controls maintained at $6 \mathrm{mmol} / \mathrm{l}$ glucose. In contrast, mTORC2-dependent AKT-473 phosphorylation in noninduced and induced DN-HNF1A- or WT-HNF1A-expressing INS-1 cells was not affected by addition of increasing concentrations of extracellular glucose (Fig. 4b-d).

\section{Induction of DN-HNFIA is associated with a reduction in} INS-1 cell size S6K1 phosphorylates the 40S ribosomal protein S6 and other proteins involved in the regulation of protein synthesis such as eukaryotic translation initiation factor 4B (eIF4B), eukaryotic elongation factor-2 kinase (eEF2K) and S6K1 specific interactor SKAR (S6K1 Aly/ REF-like substrate) [27]. Via these activities, S6K1 has been shown to be of central importance in the control of cell size $[18,27]$. We finally investigated whether the decrease in $\mathrm{mTORC} 1$ and $\mathrm{S} 6 \mathrm{~K} 1$ signalling also precipitated a reduction in INS-1 cell size during the induction of DNHNF1A. Cells were induced to express DN-HNF1A for 16, 24 and $36 \mathrm{~h}$. Flow-cytometry analyses of apoptosis activation (Annexin V staining) and cell size (forward light scatter) were performed simultaneously (Fig. 5). Annexin V detects the exposure of phosphatidylserine on the outer plasma membrane, a hallmark of apoptosis [28]. In agreement with our previous data [12, 13], we detected elevated levels of Annexin V-positive apoptotic cells from $36 \mathrm{~h}$ onwards (Fig. 5a-c). Control experiments using the general apoptosis activator staurosporine demonstrated that apoptosis induction was associated with a decrease in cell size, as cell shrinkage is a morphological hallmark of apoptosis (Fig. 5d). Therefore, only Annexin V-negative cells were evaluated for the analysis of cell-size alterations in response to DN-HNF1A. Quantification of the forward light-scatter signal in the non-apoptotic Annexin V-negative population indeed demonstrated a significant decrease in cell size after 24 and $36 \mathrm{~h}$ of DN-HNF1A expression (Fig. 5e).

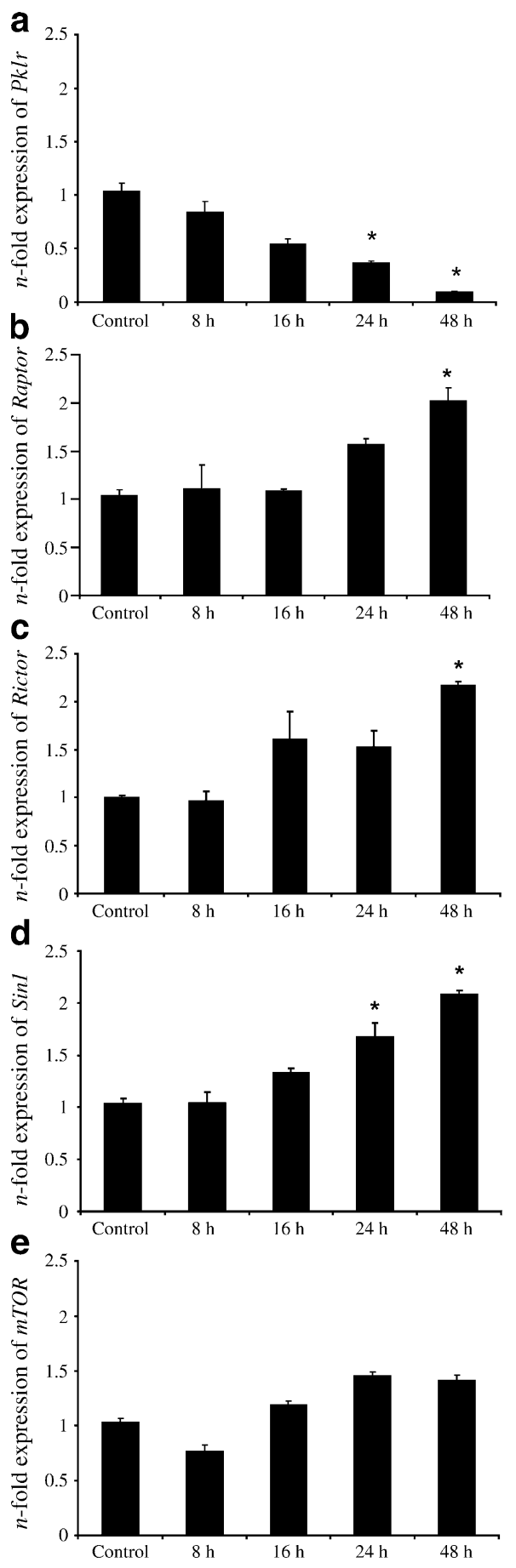

Fig. 3 Quantification of gene expression of mTorc components during DN-HNF1A induction in INS-1 cells. INS-1 cells were treated with $500 \mathrm{ng} / \mathrm{ml}$ doxycycline from 0 to $48 \mathrm{~h}$. Following treatment mRNA expression of a Pklr, b Raptor, c Rictor, d Sinl and e mTOR was examined using real-time qPCR relative to $\beta$-actin. Expression levels were normalised to control cells and data shown are the means of $n=3$ separate treatments. ${ }^{*} p<0.05$ for difference from non-induced controls. Experiments were repeated three times with similar results 


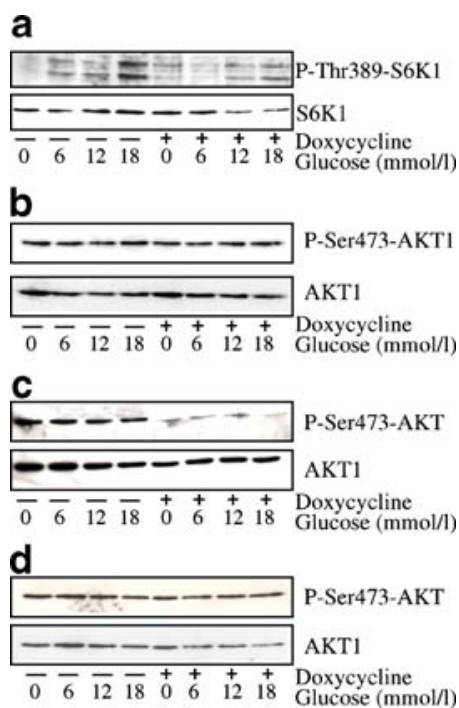

Fig. 4 Effect of extracellular glucose on mTORC1-dependent S6K and mTORC2-dependent AKT1 phosphorylation. INS-1 cells were induced to overproduce DN-HNF1A for $24 \mathrm{~h}(\mathbf{a}, \mathbf{b})$ or $48 \mathrm{~h}(\mathbf{c})$, and WT-HNF1A for $24 \mathrm{~h} \mathrm{(d)} \mathrm{in} \mathrm{the} \mathrm{presence} \mathrm{of} 6 \mathrm{mmol} / 1$ glucose, and were then exposed to the indicated glucose concentrations for $30 \mathrm{~min}$. The activation status of S6K1 or AKT1 was detected by western blotting using a Thr389- and Ser473-phosphorylation-specific antibody, respectively (P-Thr389, P-Ser473). Experiments were repeated twice with similar results

Induction of DN-HNF1A is associated with an increase in p27 mRNA and protein levels Inhibition of mTORC1 signalling leads to the hypophosphorylation of 4E-BP1 and enhanced binding to eIF4E, blocking cap-dependent translation and causing cell-cycle arrest at G1 [29]. Most eukaryotic mRNAs are translated through a cap-dependent mechanism of initiation. However, under conditions of cellular stress when cap-dependent translation is inhibited, such as nutrient deprivation and hypoxia, certain mRNAs can still be translated. Such conditions favour cap-independent translation of mRNAs containing an internal ribosome entry site (IRES) element within their 5 untranslated region [30]. p27 is a fundamental regulator of proliferation in most cell types that acts by blocking $\mathrm{G1} / \mathrm{S}$ transition through inhibition of cyclin-dependent kinase 2 (CDK2) [31]. p27 mRNA stabilisation and a switch from cap-dependent to capindependent $p 27$ mRNA translation have been identified as key pathways leading to cell-cycle arrest upon inhibition of mTORC1 [32]. We determined mRNA expression of $p 27$ in INS-1 cells induced to express DN-HNF1A by qPCR, and could detect a significant accumulation of $p 27$ mRNA $16 \mathrm{~h}$ after induction (Fig. 6a). We furthermore observed an early $(12 \mathrm{~h})$ upregulation of p27 protein levels after the induction of DN-HNF1A by western blot analysis (Fig. 6b), suggesting that the induction of DN-HNF1A establishes conditions that favour an early accumulation of the cell-cycle inhibitor p27 in INS-1 cells.

\section{Conclusion}

Our study demonstrates that signalling through mTOR and specifically through the mTORC1 pathway is disturbed early during the suppression of HNF1A function in INS-1 insulinoma cells. mTOR activity is regulated by: the availability of growth factors, such as insulin and IGF-1; the accessibility of nutrients, notably the supply of key amino acids; and AMP levels [20,33]. The role of mTOR is to integrate these diverse signals and to respond with alterations in cell metabolism, growth, proliferation, autophagy and survival [18]. Previous studies, and this study from our laboratory, have shown that prolonged, dominant negative suppression of HNF1A function causes a potent reduction in mTORC-2-dependent Ser473 phosphorylation of AKT1which coincided with the time points of maximal apoptosis [12]. Furthermore, overexpression of a constitutively active form of AKT1 was sufficient to rescue INS-1 cells from DN-HNF1A-induced apoptosis
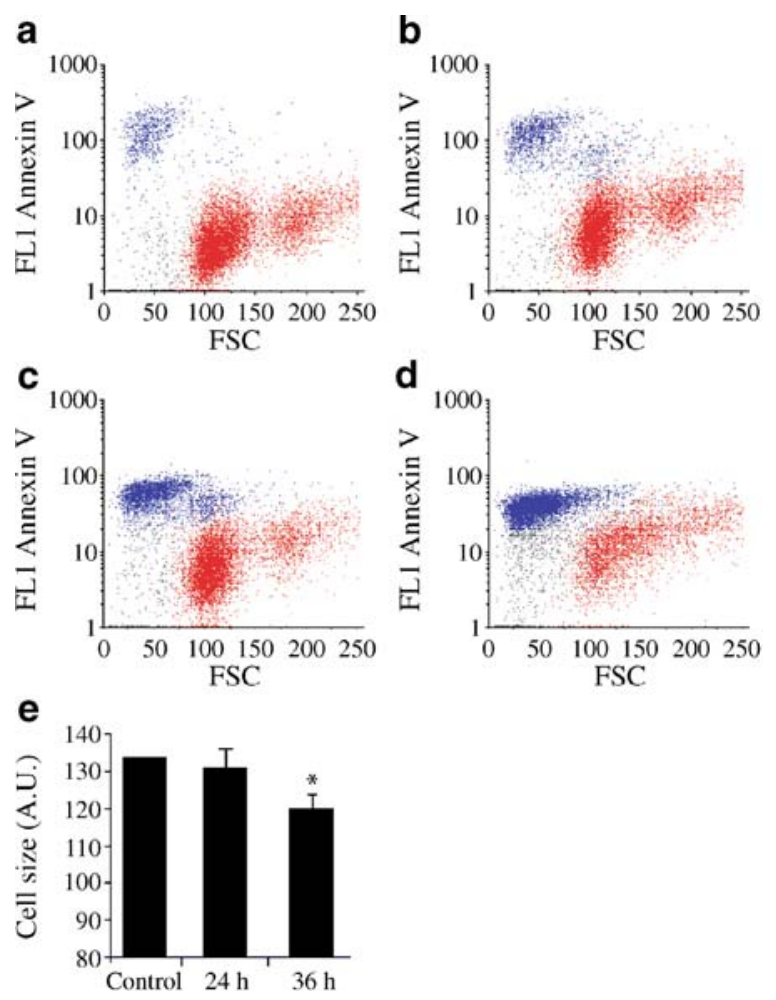

Fig. 5 Flow-cytometry analysis of cell size following DN-HNF1A induction. INS-1 cells were induced to overexpress DN-HNF1A (a non-induced; b induced $24 \mathrm{~h}$; c induced $36 \mathrm{~h}$; d STS $18 \mathrm{~h}$ ) and were then stained with Annexin V and analysed by flow cytometry using the forward scatter channel. a-d The blue population represents cells staining positive for Annexin V, an indicator of apoptosis, while cells in red represent the non-apoptotic, Annexin V-negative population that was used for cell-size quantification. e Statistical analysis of the timedependent decrease in cell size. Data are means $+\mathrm{SD} ; * p<0.05$, difference from non-induced controls. A.U., arbitrary units; STS, staurosporine 
a

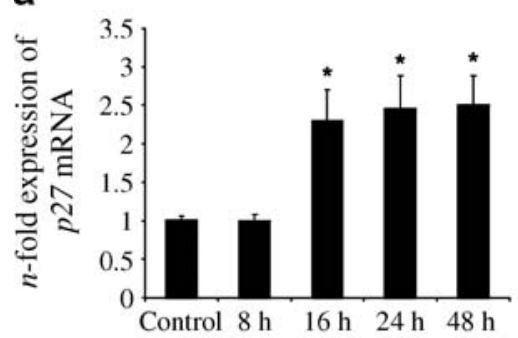

b

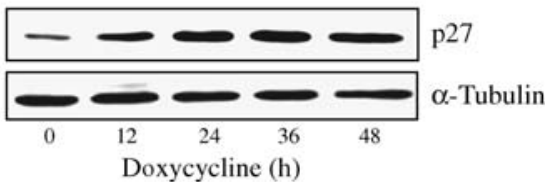

Fig. 6 Reduction in mTORC1 signalling is associated with increased mRNA and protein levels of the cell-cycle inhibitor $p 27$. INS-1 cells were induced to overproduce DN-HNF1A for the indicated time periods. a Quantification of p27 mRNA using real-time quantitative PCR. Data shown are the means of $n=3$ separate treatments. The experiment was repeated three times with similar results. ${ }^{*} p<0.05$, difference from non-induced controls. b The expression of p27 was detected by western blotting using a p27-specific antibody. A duplicate experiment yielded similar results

[12]. Hence, mTORC2 and AKT1 inactivation appear to mediate aspects of long-term alterations such as apoptosis activation in response to DN-HNF1A.

In the present study, we noted an early inactivation of mTORC1 signalling in response to DN-HNF1A expression, as demonstrated by the inhibition of Thr389 phosphorylation of S6K1 and Ser65 phosphorylation of 4E-BP1. mTORC1 signalling via phosphorylation and activation of $\mathrm{S} 6 \mathrm{~K}$ is an important regulator of cell size [18]. S6K1 phosphorylates the ribosomal protein S6 leading to selective translation of $5^{\prime}$ terminal oligopyrimidine tract (TOP) mRNAs. These oligopyrimidine-containing mRNAs encode for components of the translational apparatus including ribosomal proteins and translation elongation factors, thereby enhancing the translational capacity. It is interesting to mention in this context that a recent microarray analysis has also shown that DN-HNF1A expression decreases the transcription of several ribosomal proteins [34]. Targets of S6K1 other than ribosomal protein S6 have been identified, such as the transcription factor cAMP responsive element modulator (CREM)- $\tau$, the RNA splicing/export factor cap binding protein 80 (CBP80) and the eukaryotic translation elongation factor 2 (eEF2) [35], which may mediate additional activities of S6K1 that were hitherto partially unknown. Loss of $S 6 \mathrm{kl}$ in Drosophila is semi-lethal, and a markedly reduced body size was seen in the surviving adults [36]. This reduction in body size was caused by a decrease in cell size rather than a decrease in the actual cell number [36]. In mice, deletion of $S 6 k 1$ was not shown to be lethal; however, the $S 6 \mathrm{kl}$-deficient animals had a signifi- cantly reduced birthweight. Interestingly, the mice also had reduced circulating insulin levels resulting from a reduction in beta cell size [37]. In our studies we observed a timedependent reduction in INS- 1 cell size during dominant negative suppression of HNF1A function prior to the onset of apoptosis, thus suggesting that an early decrease in mTORC1 signalling leads to early reduction in cell size. A decrease in beta cell size (in addition to an overall decrease in beta cell mass) has also been suggested to occur in animal models of HNF1A-MODY [38]. It is tempting to speculate that such a decrease would also occur in HNF1AMODY individuals. Since the insulin secretion rate is highly sensitive to alterations in the beta cell surface area [39], even small alterations in cell size could contribute to the reported insulin secretory defects in HNF1A-MODY [9].

mTOR has also been shown to hyperphosphorylate at several sites causing the liberation of eIF4E from 4E-BP and the association of eIF4E with both capped mRNA and eukaryotic-initiation-factor-4G (eIF4G). This leads to the assembly of the translation-initiation complex at the mRNA $5^{\prime}$ cap, promoting $5^{\prime}$ cap-dependent protein synthesis. A role for the switch from cap-dependent to cap-independent translation has been identified as a key signalling pathway in cell-cycle and apoptosis control [32, 40]. We could detect an early upregulation of $p 27$ mRNA and protein levels in response to DN-HNF1A expression, prior to AKT1 inactivation [12]. Inhibition of $\mathrm{mTORC} 1$ has been shown to stabilise p27 mRNA and to promote cap-independent translation of $p 27$ mRNA, hence both mRNA and protein levels may be upregulated independent of gene activation [31]. A recent study in human breast-cancer cell lines has shown that inhibition of mTORC1 with rapamycin also leads to reduced p27 protein degradation via decreased expression of the ubiquitin ligase subunit $S k p 2$ [41]. Hence the early increase in $\mathrm{p} 27$ protein levels may also be due to a decrease in p27 degradation. However, we cannot fully exclude that $p 27$ gene activation via AKT1 inactivation and subsequent forkhead box O3a (FOXO3a) activation may contribute to the early upregulation of $p 27 \mathrm{mRNA}$ and protein levels observed in this and other studies. Together with previous reports demonstrating elevated p27 levels in cell culture and animal models of HNF1A-MODY [34, 42], our data therefore suggest that mTORC1 inhibition may also contribute to an early decrease in beta cell mass in HNF1A-MODY by inhibiting cell proliferation.

What could be the potential mechanisms responsible for the early mTORC1 vs late mTORC2 inactivation in response to DN-HNF1A? Our data demonstrate that gene expression changes of mTorc constituents appear to play no significant role in this differential inactivation. AKT1 activates mTORC1 and eventually S6K1 by a direct action on PRAS40 and via TSC1-TSC2 and RHEB GTPase [16]. 
Overexpression of a constitutively active form of Akt1 has been shown to be associated with an increased S6K activity [43]. It has also been shown that targeted overexpression of constitutively active Akt1 in transgenic mice induced a striking increase in beta cell mass caused by an increase in both beta cell number and size [44]. However, our study demonstrated different dephosphorylation kinetics in response to DN-HNF1A expression, suggesting that AKT1 inactivation may not be the primary mechanism of mTORC1 and S6K1 inactivation in this model. Importantly, cellular ATP depletion has also been reported to occur early during the induction of DN-HNF1A in INS-1 cells [22].

Energy depletion triggers the activation of AMP-activated protein kinase (AMPK) which, in turn, phosphorylates the TSC1-TSC2 complex leading to an inhibition of mTORC1 [15]. We report here that although the basal phosphorylation of S6K1 was significantly reduced after induction of DNHNF1A, mTORC1-dependent S6K1 phosphorylation was still partially responsive to high glucose. This glucosestimulated activation of S6K1 is possibly mediated by increased ATP supply and reduced AMPK activation. Dominant-negative suppression of HNF1A has also been shown to reduce glucose-induced $\mathrm{Ca}^{2+}$ signalling in INS-1 cells [21], an event that may also contribute to the early inactivation of S6K1 and its partial rescue by high glucose, as increased intracellular $\mathrm{Ca}^{2+}$ concentrations have been shown to activate S6K1 but not AKT1 [45]. MAP kinase signalling pathways in particular are activated by increasing glucose and $\mathrm{Ca}^{2+}$ concentrations $[46,47]$. A variety of proline-directed kinases including extracellular-signal-regulated kinase (ERK)1/2 and cell division cycle 2, G1 to S and G2 to $\mathrm{M}(\mathrm{CDC} 2)$ are capable of phosphorylating S6K1 in the C-terminal autoinhibitory pseudosubstrate domain of these sites in vitro [48], allowing control of the activation of S6K1 independently of the PI3K/PDK1 pathway. While multiple signalling pathways can act upon mTORC1 to regulate signalling through this complex, the mTORC2-signalling complex appears to be a more robust biological system. Indeed, mTORC1 appears to predominantly mediate shortterm adaption to changes such as growth-factor supply, nutrient supply, hypoxia or stress, while mTORC2 is concerned with long-term changes such as spatial organisation and apoptosis. In our model, glucose did not affect mTORC2, and mTORC2-dependent AKT1 phosphorylation was not reduced until $48 \mathrm{~h}$, as previously reported [12]. Reduced expression of Ins I, IGFI and Akt1 genes in response to DN-HNF1A [12, 21, 33] may eventually lead to an inability to sustain high levels of active AKT1.

In summary, our data demonstrate that mTOR signalling via $\mathrm{mTORC} 1$ is disturbed early during the suppression of HNF1A function, and suggest that decreased signalling through mTORC1 may contribute to a disturbance of cell size and cell-cycle regulation in HNFF1A-MODY.
Acknowledgements We thank H. Wang and C. Wollheim for the INS-1 clones and R. Cortese for kindly supplying the HNF1A antibody. This work was supported by a grant from the Health Research Board (RP/2004/220) to M. M. Byrne, and a grant from the Higher Education Authority (Program of Human Genomics) to J. H. M. Prehn.

Duality of interest The authors declare that there is no duality of interest associated with this manuscript.

\section{References}

1. Fajans SS, Bell GI, Polonsky KS (2001) Molecular mechanisms and clinical pathophysiology of maturity-onset diabetes of the young. N Engl J Med 345:971-980

2. Frayling TM, Bulamn MP, Ellard S et al (1997) Mutations in the hepatocyte nuclear factor-1alpha gene are a common cause of maturity-onset diabetes of the young in the U.K. Diabetes 46:720-725

3. Ellard S, Colclough K (2006) Mutations in the genes encoding the transcription factors hepatocyte nuclear factor 1 alpha (HNF1A) and 4 alpha (HNF4A) in maturity-onset diabetes of the young. Human Mutat 27:854-869

4. Yamagata K, Oda N, Kaisaki PJ et al (1996) Mutations in the hepatocyte nuclear factor-1alpha gene in maturity-onset diabetes of the young (MODY3). Nature 384:455-458

5. Blumenfeld M, Maury M, Chouard T, Yaniv M, Condamine H (1991) Hepatic nuclear factor 1 (HNF1) shows a wider distribution than products of its known target genes in developing mouse. Development 113:589-599

6. Odom DT, Zizlsperger N, Gordon DB et al (2004) Control of pancreas and liver gene expression by HNF transcription factors. Science 303:1378-1381

7. Holmkvist J, Cervin C, Lyssenko V et al (2006) Common variants in HNF-1 alpha and risk of type 2 diabetes. Diabetologia 49:2882-2891

8. Pontoglio M, Sreenan S, Roe M et al (1998) Defective insulin secretion in hepatocyte nuclear factor 1alpha-deficient mice. J Clin Invest 101:2215-2222

9. Byrne MM, Sturis J, Menzel S et al (1996) Altered insulin secretory responses to glucose in diabetic and nondiabetic subjects with mutations in the diabetes susceptibility gene MODY3 on chromosome 12. Diabetes 45:1503-1510

10. Wang H, Hagenfeldt-Johansson K, Otten LA, Gauthier BR, Herrera PL, Wollheim CB (2002) Experimental models of transcription factor-associated maturity-onset diabetes of the young. Diabetes 51(Suppl 3):S333-S342

11. Wang H, Antinozzi PA, Hagenfeldt KA, Maechler P, Wollheim CB (2000) Molecular targets of a human HNF1 alpha mutation responsible for pancreatic beta-cell dysfunction. EMBO J 19:4257-4264

12. Wobser H, Bonner C, Nolan JJ, Byrne MM, Prehn JH (2006) Downregulation of protein kinase B/Akt-1 mediates INS-1 insulinoma cell apoptosis induced by dominant-negative suppression of hepatocyte nuclear factor-1alpha function. Diabetologia 49:519-526

13. Wobser H, Dussmann H, Kogel D et al (2002) Dominant-negative suppression of HNF-1 alpha results in mitochondrial dysfunction, INS-1 cell apoptosis, and increased sensitivity to ceramide-, but not to high glucose-induced cell death. J Biol Chem 277:6413-6421

14. Heitman J, Movva NR, Hall MN (1991) Targets for cell cycle arrest by the immunosuppressant rapamycin in yeast. Science 253:905-909

15. Inoki K, Zhu T, Guan KL (2003) TSC2 mediates cellular energy response to control cell growth and survival. Cell 115:577-590 
16. Sancak Y, Thoreen CC, Peterson TR et al (2007) PRAS40 is an insulin-regulated inhibitor of the mTORC1 protein kinase. Mol Cell 25:903-915

17. Guertin DA, Sabatini DM (2007) Defining the role of mTOR in cancer. Cancer Cell 12:9-22

18. Wullschleger S, Loewith R, Hall MN (2006) TOR signaling in growth and metabolism. Cell 124:471-484

19. Jacinto E, Facchinetti V, Liu D et al (2006) SIN1/MIP1 maintains rictor-mTOR complex integrity and regulates Akt phosphorylation and substrate specificity. Cell 127:125-137

20. Sarbassov DD, Guertin DA, Ali SM, Sabatini DM (2005) Phosphorylation and regulation of $\mathrm{Akt} / \mathrm{PKB}$ by the rictor-mTOR complex. Science 307:1098-1101

21. Huang J, Dibble CC, Matsuzaki M, Manning BD (2008) The TSC1-TSC2 complex is required for proper activation of mTOR complex 2. Mol Cell Biol 28:4104-4115

22. Wang H, Maechler P, Hagenfeldt KA, Wollheim CB (1998) Dominant-negative suppression of HNF-1alpha function results in defective insulin gene transcription and impaired metabolismsecretion coupling in a pancreatic beta-cell line. EMBO J 17:6701-6713

23. Nicosia A, Monaci P, Tomei L et al (1990) A myosin-like dimerization helix and an extra-large homeodomain are essential elements of the tripartite DNA binding structure of LFB1. Cell 61:1225-1236

24. Rozen S, Skaletsky H (2000) Primer3 on the WWW for general users and for biologist programmers. Methods Mol Biol 132:365-386

25. Gingras AC, Raught B, Sonenberg N (2001) Regulation of translation initiation by FRAP/mTOR. Genes Dev 15:807-826

26. Holz MK, Blenis J (2005) Identification of S6 kinase 1 as a novel mammalian target of rapamycin (mTOR)-phosphorylating kinase. J Biol Chem 280:26089-26093

27. Ruvinsky I, Meyuhas O (2006) Ribosomal protein S6 phosphorylation: from protein synthesis to cell size. Trends Biochem Sci $31: 342-348$

28. van Engeland M, Nieland LJ, Ramaekers FC, Schutte B, Reutelingsperger CP (1998) Annexin V-affinity assay: a review on an apoptosis detection system based on phosphatidylserine exposure. Cytometry 31:1-9

29. Wang X, Beugnet A, Murakami M, Yamanaka S, Proud CG (2005) Distinct signaling events downstream of mTOR cooperate to mediate the effects of amino acids and insulin on initiation factor 4E-binding proteins. Mol Cell Biol 25:2558-2572

30. Pause A, Belsham GJ, Gingras AC et al (1994) Insulin-dependent stimulation of protein synthesis by phosphorylation of a regulator of 5'-cap function. Nature 371:762-767

31. Sherr CJ, Roberts JM (1999) CDK inhibitors: positive and negative regulators of G1-phase progression. Genes Dev 13:1501-1512

32. Miskimins WK, Wang G, Hawkinson M, Miskimins R (2001) Control of cyclin-dependent kinase inhibitor p27 expression by cap-independent translation. Mol Cell Biol 21:4960-4967

33. Gwinn DM, Shackelford DB, Egan DF et al (2008) AMPK phosphorylation of raptor mediates a metabolic checkpoint. Mol Cell 30:214
34. Yang Q, Yamagata K, Fukui K et al (2002) Hepatocyte nuclear factor-1alpha modulates pancreatic beta-cell growth by regulating the expression of insulin-like growth factor-1 in INS-1 cells. Diabetes 51:1785-1792

35. Hannan KM, Brandenburger Y, Jenkins A et al (2003) mTORdependent regulation of ribosomal gene transcription requires S6K1 and is mediated by phosphorylation of the carboxy-terminal activation domain of the nucleolar transcription factor UBF. Mol Cell Biol 23:8862-8877

36. Montagne J, Stewart MJ, Stocker H, Hafen E, Kozma SC, Thomas G (1999) Drosophila S6 kinase: a regulator of cell size. Science 285:2126-2129

37. Ruvinsky I, Sharon N, Lerer T et al (2005) Ribosomal protein S6 phosphorylation is a determinant of cell size and glucose homeostasis. Genes Dev 19:2199-2211

38. Hagenfeldt-Johansson KA, Herrera PL, Wang H, Gjinovci A, Ishihara H, Wollheim CB (2001) Beta-cell-targeted expression of a dominant-negative hepatocyte nuclear factor-1 alpha induces a maturity-onset diabetes of the young (MODY)3-like phenotype in transgenic mice. Endocrinology 142:5311-5320

39. Giordano E, Cirulli V, Bosco D, Rouiller D, Halban P, Meda P (1993) B-cell size influences glucose-stimulated insulin secretion. Am J Physiol 265:C358-364

40. Luo Y, Marx SO, Kiyokawa H, Koff A, Massague J, Marks AR (1996) Rapamycin resistance tied to defective regulation of p27Kip1. Mol Cell Biol 16:6744-6751

41. Shapira M, Kakiashvili E, Rosenberg T, Hershko DD (2006) The mTOR inhibitor rapamycin down-regulates the expression of the ubiquitin ligase subunit Skp2 in breast cancer cells. Breast Cancer Res 8:R46

42. Luco RF, Maestro MA, del Pozo N, Philbrick WM, de la Ossa PP, Ferrer J (2006) A conditional model reveals that induction of hepatocyte nuclear factor-1alpha in Hnflalpha-null mutant betacells can activate silenced genes postnatally, whereas overexpression is deleterious. Diabetes 55:2202-2211

43. Shioi T, McMullen JR, Kang PM et al (2002) Akt/protein kinase B promotes organ growth in transgenic mice. Mol Cell Biol 22:27992809

44. Fatrai S, Elghazi L, Balcazar N et al (2006) Akt induces beta-cell proliferation by regulating cyclin D1, cyclin D2, and p21 levels and cyclin-dependent kinase-4 activity. Diabetes 55:318-325

45. Conus NM, Hemmings BA, Pearson RB (1998) Differential regulation by calcium reveals distinct signaling requirements for the activation of Akt and p70S6k. J Biol Chem 273:4776-4782

46. Frodin M, Sekine N, Roche E et al (1995) Glucose, other secretagogues, and nerve growth factor stimulate mitogen-activated protein kinase in the insulin-secreting beta-cell line, INS-1. J Biol Chem 270:7882-7889

47. Khoo S, Cobb MH (1997) Activation of mitogen-activating protein kinase by glucose is not required for insulin secretion. Proc Natl Acad Sci U S A 94:5599-5604

48. Papst PJ, Sugiyama H, Nagasawa M, Lucas JJ, Maller JL, Terada N (1998) Cdc2-cyclin B phosphorylates p70 S6 kinase on Ser411 at mitosis. J Biol Chem 273:15077-15084 